\title{
EVIDENCIA DE LAS DIFERENCIAS DE EXPECTATIVAS EN AUDITORIA EN MERCADOS BURSÁTILES DE REDUCIDA DIMENSIÓN: EL CASO PORTUGUÉS
}

\author{
EVIDENCE ON AUDIT EXPECTATION GAPS IN SMALL \\ CAPITAL MARKETS: THE CASE OF PORTUGAL
}

BRUNO JOSÉ MACHADO DE ALMEIDA

Profesor Adjunto (equiparado) da Escola Superior de

Gestão de Tomar do Institüto Politécnico de Tomar

E-mail: brunojmalmeida@clix.pt

\author{
CLARA ISABEL MUÑOZ COLOMINA \\ Profesora Doctora do Departamento de Economía Financiera y \\ Contabilidad II da Facultad de Ciencias Económicas y Empresariales \\ da Universidade Complutense de Madrid \\ E-mail: cimunnoz@ccee.ucm.es
}

\section{RESUMEN}

El principal objetivo de esta investigación es analizar, en un pequeño mercado de capitales, como es el de Portugal, si existen diferencias de expectativas en auditoria y cuales son sus componentes principales. Para ese efecto se há cuestionado a auditores e profesores de contabilidad e auditoria en relación a diversos asuntos que influyen e incrementan las diferencias de expectativas en auditoria. Por otro lado, también se cuestiona a los usuarios de la información financiera en Portugal, intentando captar cuales son sus percepciones del papel de la auditoria contemporánea, y sus respuestas son comparadas con las opiniones de usuarios de los países anglosajones. Se concluye que las expectativas de los usuarios de la información en Portugal son semejantes a la de los países anglosajones. El fraude es el principal tópico en las diferencias de expectativas. Por.otro lado, el modelo actual de informes de auditoria en Portugal no es suficiente claro para que los usuarios puedan percibir cual es el alcance y el trabajo desarrollado por el auditor en la obtención de sus conclusiones.

Palabras-llave: Auditoria. Diferencias de expectativas. Usuarios. Auditores.

\section{ABSTRACT}

The main goal of this paper is to analyze if, in a small capital market such as the Portuguese, the audit expectation gap exits and which are its major components. We have enquired auditors and University accounting and auditing professors regarding several subjects that influence and increase the audit expectation gap. We have also questioned the Portuguese users of financial information regarding their perception of the role of today's auditing, to assay their opinion with studies made in Anglo-Saxon countries. We have reached the conclusion that Portuguese financial statements users' expectations are similar to the ones of bigger capital markets. As a matter of fact, fraud is the main cause for the existence of the audit expectation gap, nevertheless, Portuguese financial statements users also indicate that the actual auditor's report model in Portugal is clearly insufficient to comprehend the objectives and work that auditors have done to reach their conclusions.

Keywords: Audit. Expectation gap. Users. Auditors. 


\section{INTRODUCCIÓN}

Las diferencias de expectativas en auditoría han venido afectando a los profesionales de la misma desde hace muchos años, siendo responsables por el ambiente de crítica y litigio, movido por los tribunales, por los políticos, por la prensa y por la sociedad contra los auditores en todo lo concerniente a su calidad y desempeño. Como bien es reconocido por Godsell (1992, p. 25-28), existe entre los usuarios de la información financiera la opinión generalizada de que cualquier persona que tenga un determinado interés en una empresa debe ser capaz de confiar en las cuentas auditadas como garantía de solvencia y viabilidad de la misma. Por ese motivo, al llegar a ser de conocimiento público, sin cualquier aviso previo, el hecho de que una empresa se encuentra en serias dificultades financieras, se asiste inmediatamente al intento de hacer responsable a alguien de esa situación y ese alguien es el auditor.

A pesar de no ser un tema reciente, las diferencias de expectativas vienen ocupando, cada vez más, un lugar cimero en el debate sobre el futuro de la auditoría y sobre su papel ante la sociedad. Los sucesivos y recientes escándalos financieros - Enron, WorlCom, Xerox, Parmalat, entre otros -, que han asolado el mercado de capitales y a la sociedad, han venido a reforzar entre el público la idea de que la auditoría no está respondiendo a las expectativas de la sociedad. En efecto, esta cuestión se está transformando en una amenaza y convirtiendo a los profesionales de la auditoría en un blanco muy vulnerable, hasta el extremo de llegar a ponerse en tela de juicio su papel en la sociedad actual. La sociedad precisa tener confianza en la calidad, integridad y fiabilidad de la información financiera divulgada por los gestores y administradores de las empresas $y$, es, con esa finalidad, por lo que les confía a los auditores el papel de verificar si dicha información es el reflejo fiel de una imagen adecuada de la empresa.

Esta problemática está localizada, por una parte, entre un cuadro de usuarios de la información financiera que no comprende la responsabilidad de los auditores, la opinión manifestada en el informe de los auditores, los límites inherentes a la propia auditoría, así como tampoco entiende el tipo de información trasmitida y, por otra parte, está localizada en el desarrollo práctico de la auditoría que, al basarse en el cuadro de la corriente tradicional de la auditoría, estructura la actividad de los auditores en la consecución de una minimización del riesgo y en el estricto cumplimiento de las normas técnicas de la profesión. Emerge, asimismo, la posición que adoptan los sujetos pasivos de la auditoría que se quejan de su imposición y de la relación costo / beneficio y que alegan que la auditoría no tiene más que inconvenientes y ningún fruto concreto. Se coloca, también, en tela de juicio, el valor agregado generado por la auditoría al limitarse ésta a una actividad de análisis, verificación y validación de documentos solamente contables, cuando lo que la sociedad, cada vez más y con un nivel de exigencia mayor, considera que la profesión debería abarcar aspectos más amplios que el simple concepto de imagen fiel, verdadera y apropiada, que, por su propio reduccionismo, no contribuye a un conocimiento holístico de la actividad empresarial auditada.

El objetivo global del estudio consiste en el encuadramiento de la auditoría y la investigación del papel que cumple en la dinámica de la sociedad actual, que ha generado, de forma exógena o endógena, una tendencia para la regulación a través de los designados poderes de compensación que sirven de contrapeso a poderes como la riqueza, la seguridad, la productividad, etc. con valores sociales y culturales. Como objetivo primordial de esta investigación, Se pretende analizar de forma integrada los diversos componentes de la diferencia de expectativas en auditoría y aplicarlos a la realidad portuguesa, donde todavía hoy no ha sido desarrollado ningún estudio en este campo, y compararlos con el panorama internacional.

\section{LAS DIFERENCIAS DE EXPECTATIVAS EN AUDITORIA}

Han sido realizados varios estudios empíricos sobre la problemática de las diferencias de expectativas en auditoría que, teniendo como objetivo el poner a descubierto los factores explicativos de esas diferencias, han analizado no sólo el papel del auditor y la forma de entender sus responsabilidades, sino también su trabajo en sí mismo.

Basándose, esencialmente, en cuestionarios realizados entre los auditores y el público, Baron et al. (1977, p. 243-250) examinaron la amplitud de las responsabilidades de los auditores en la detección de errores materialmente relevantes, de irregularidades y de actos ilegales. Estos autores intentaron averiguar si existían diferencias entre los auditores y los usuarios de la información financiera, en lo que se refería a cómo entendían ambos los deberes de detección y de informe que tiene el auditor. Llegaron a la conclusión de que los usuarios de la información financiera consideran que los auditores tienen una mayor responsabilidad por la detección de errores, irregularidades y otros actos ilegales, que la que, realmente, los auditores Ilegan a reconocer.

Lowe (1980, p. 65-70) examinó las diferencias de expectativas en Australia. El autor abordó la cuestión del aumento de las responsabilidades relacionadas con la detección de errores, irregularidades y actos ilegales de acuerdo con la forma de entender este asunto que tenían auditores y no auditores. Dedujo que los dos grupos difieren, significativamente, en relación con la percepción del aumento de las responsabilidades de los auditores en lo que se refiere a la detección de errores y comunicación de materias en las 
áreas bajo su responsabilidad: las diferencias entre los dos grupos eran reales.

También, en Gran Bretaña, Humphrey et al. (1993, p. 359-411) examinaron las diferencias de expectativas, descubriendo la percepción del público en relación con las expectativas que tenía, a través de la formulación de una serie de cuestiones: ¿Cuál es y cuál debería ser el papel del auditor? ¿Cuáles deberían ser las prohibiciones y reglamentos de las empresas de auditoría? ¿Qué decisiones se debe esperar que el auditor tome? El estudio reveló que existe una significativa diferencia entre los auditores y los usuarios de la información financiera, en lo que se refiere a sus perspectivas sobre la naturaleza de la auditoría. Los resultados confirmaron que las diferencias de expectativas existen, especialmente, en el ámbito de la función de la auditoría y en la percepción del desempeño del trabajo del auditor y que los componentes críticos de las diferencias de expectativas comprenden diversos aspectos: el papel del auditor en la detección de fraudes, la independencia del auditor, la ampliación de las responsabilidades del auditor y los aspectos relacionados con la forma de conducir una auditoría.

Más recientemente, en 2001, McEnroe y Martens (2001, p. 345-358) han llevado a cabo una investigación para analizar la percepción de los auditores en relación con las diferencias de expectativas y han llegado a la conclusión de que las diferencias de expectativas son una realidad, porque los inversionistas colocan expectativas muy elevadas en lo que se refiere a la seguridad que la auditoría debe proporcionar en diversas áreas, entre las que se destacan: aspectos relacionados con los informes comunicados por el auditor, control interno, fraude y actos ilegales. Han concluido, de forma muy expresiva, que lo que los inversionistas esperan de los auditores es que sirvan de «perros de guarda».

Se puede decir que las pruebas de la existencia de las diferencias de expectativas en auditoría son sustanciales.

El Figura 10 proporciona una visión amplia de la estructura del problema de las diferencias de expectativas:

Se puede observar que las diferencias de expectativas tienen dos grandes componentes de base: la diferencia de razonabilidad y la diferencia en el desempeño; ésta última, a su vez, dividida en normas inadecuadas y desempeño inadecuado

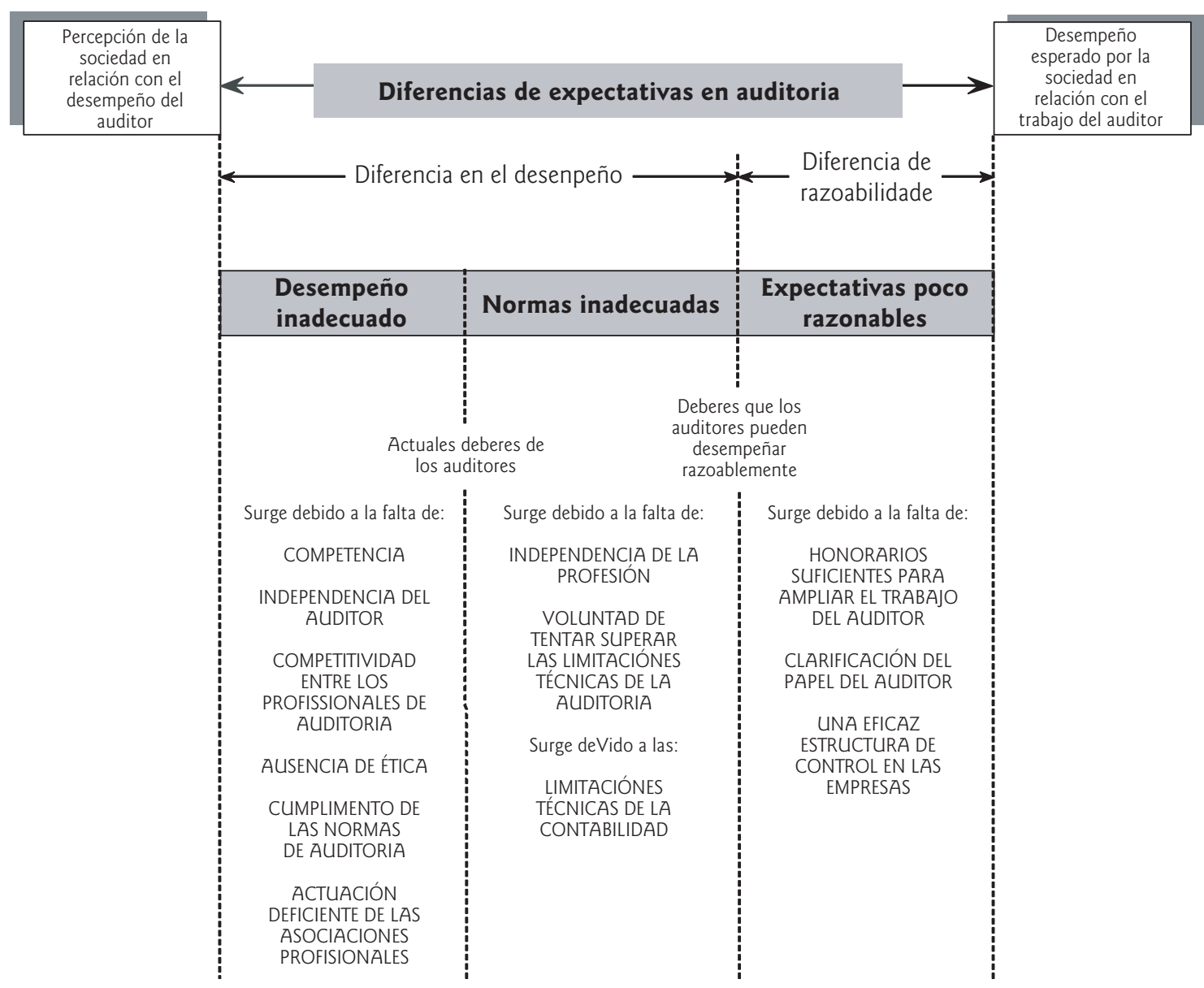

Figura 1 Los componentes estructurales de las diferencias de expectativas 


\section{LAS DIFERENCIAS DE EXPECTATIVAS EN PORTUGAL: SELECCIÓN DEL MUESTREO}

El procedimiento utilizado para concretizar el estudio sobre la problemática de las diferencias de expectativas en Portugal se materializó en la elaboración de dos tipos de cuestionarios. El primero estaba orientado hacia los técnicos profesionales (Censores Jurados de Cuentas, Auditores internos y Auditores del Tribunal del Cuentas) y hacia los profesores y el segundo se envió a analistas financieros, a empresas con cotización bursátil y a aseguradoras que operan en Portugal.

En relación con el primero se plantearon diversas cuestiones relacionadas con los siguientes grupos técnicos: normas de auditoría, comportamiento de los auditores, comportamiento de los gestores, limitaciones técnicas de la auditoría, gobierno de las sociedades, estructura de control, mercado de auditoría, actuación de las asociaciones de los colegios profesionales, comportamiento de los usuarios de la información financiera, limitaciones de la contabilidad y cuestiones referentes a la percepción que la sociedad tiene de la auditoría. Cada uno de los conjuntos antes descritos contiene varias afirmaciones a las que los encuestados deben responder si están, o no, de acuerdo con ellas.

El segundo tenía como objetivo conocer cuáles son las expectativas de las personas cuestionadas en relación con el trabajo de auditoría, analizando si éstas podrían ser razonablemente incluidas en el trabajo del auditor o si, por el contrario, se corresponden con expectativas imposibles de alcanzar o de satisfacer.

La razón justificativa del envío de dos tipos de cuestionarios diferentes se basa en el hecho de que al primero, como cuerpo profesional, le son colocadas cuestiones eminentemente técnicas a las que sólo profesionales del área o profesores de las respectivas materias sabrían dar respuesta, mientras que de los segundos se quiere conocer su estado de opinión, no abordando temas específicos de auditoría como ocurría en el primer cuestionario, sino el interés se centra en conocer cuáles son sus expectativas sobre los profesionales de auditoría, comparar esas opiniones con las normas y con las promulgaciones profesionales.

El estudio empírico realizado fue desarrollado a través del envío de cuestionarios a los diferentes grupos participantes, contadose para ese efecto con la colaboración de los Colegios Oficiales y Asociaciones profesionales que fueron quienes dieron curso y encaminaron los cuestionarios hacia sus destinatarios. Para ello, se solicito la colaboración de los siguientes organismos: Colegio Oficial de Censores Jurados de Cuentas, Asociación Portuguesa de Analistas Financieros, Instituto Portugués de Auditores Internos Tribunal de Cuentas y varias Universidades e Institutos Superiores Politécnicos portugueses. La expedición y recepción de los cuestionarios se fue realizando entre los meses de Enero y Agosto de 2005.

La muestra escogida para llevar a cabo este estudio es seleccionada entre varias clases que se interrelacionan con la auditoría, es decir, los Censores Jurados de Cuentas (ROC), los auditores internos (Al), los auditores del Tribunal de Cuentas (ATC), los profesores de Enseñanza Superior a cuyo cargo se encuentran en sus respectivos centros educativos las asignaturas de Contabilidad y Auditoría, los Analistas Financieros y las empresas con cotización bursátil (índice PSI-Geral) y empresas aseguradoras.

Los cuestionarios enviados y recibidos para cada uno de los grupos han sido (Tabla $1 \boldsymbol{\nabla}$ ):

Con los cuestionarios recibidos de cada grupo se procuró responder à las siguientes cuestiones:

- iCual es la componente de las diferencias de expectativas, que para cada grupo, más contribuí para su existencia?

- ¿Cual los aspectos diferenciadores entre los profesores y los auditores en relación à la diferencia de expectativas?

- ¿Cual es la opinión de los usuarios de la información financiera sobre el papel, ámbito y limitaciones de la auditoria?

\section{COMPONENTES DE LAS DIFERENCIAS DE EXPECTATIVAS QUE MÁS CONTRIBUYEN PARA SU EXISTENCIA}

En este punto es decido comparar la opinión emitida por los profesores, por los ROC's, por los Al y por los
ATC's en lo que se relaciona con los componentes del modelo que más han contribuido para la existencia de las

Tabla 1 | Cuestionarios enviados y recebidos

\begin{tabular}{l|c|c|c} 
& Población encuestada & Encuestas recibidas & \% de respuestas \\
\hline ROC's & 766 & 212 & $28 \%$ \\
\hline Al & 300 & 51 & $17 \%$ \\
\hline ATC & 39 & 25 & $64 \%$ \\
\hline Prof's & 170 & 159 & $94 \%$ \\
\hline Emp. & 54 & 21 & $39 \%$ \\
\hline AF & 220 & 53 & $24 \%$ \\
\hline & 1549 & 521 &
\end{tabular}


diferencias de expectativas, tal como es presentado en la figura 1.

Así, se asocia algunos grupos de cuestiones que se encuadraban por su carácter en cada uno de los componentes identificados de las diferencias de expectativas, procurando comparar, separadamente, la sensibilidad de los encuestados en torno a cada componente.

Cada grupo de cuestiones agregadas es asociado a un componente, atribuyendo a cada uno un peso idéntico, por lo que se escoge un número semejante de cuestiones para evaluar cada componente.

Así, se lleva a cabo la siguiente correspondencia (Tabla 2 ( )

En lo realcionado a las diferencias de razonabilidad se procede a dividir en dos sus causas generadoras: la organización y la sociedad, procurando determinar a cuál de estos componentes es al que los encuestados le atribuyen un mayor peso.

Las variables conceptuales (normas inadecuadas, desempeño inadecuado y expectativas poco razonables relacionadas con la organización y con la sociedad) fueron medidas a través de los diversos ítems que constituyen las preguntas del cuestionario.

Se considera una medida adecuada aquélla que tiene una fiabilidad adecuada, es decir, un coeficiente interno alfa de Cronbach ${ }^{1}$ superior a 0,52 y unidimensional (es decir, que defina un solo factor en un análisis factorial).

Como se puede verificar por la tabla que se expone más adelante, las variables que componen las diferencias de desempeño y las diferencias de razonabilidad presentan una fiabilidad adecuada, una vez que el alfa de Cronbach para estas variables es superior a 0,5 .

Sin embargo, para cada una de las variables hemos tenido que retirar algunas cuestiones, debido a que éstas no tenían un poder explicativo, pues el alfa de Cronbach aumentaba considerablemente en el caso de eliminarlas; esto fue realizado, relativamente a la primera variable, a las cuestiones 02.01 y 02.04 (el alfa de Cronbach pasó de 0,693 para 0,774 y después para 0,785 ) (Tabla $3 \mathbf{\nabla}$ ).

Después de retirarse las cuestiones referidas en el cuadro anterior, se suman todos los valores para construir las variables «desempeño inadecuado», «normas inadecuadas», «expectativas poco razonables relacionada con la organización» y "expectativas poco razonables relacionadas con la sociedad».

Ya que se suman todos los valores para construir las variables, es necesario saber si éstas son unidimensionales, es decir, si están midiendo apenas un componente o si, por el contrario, están midiendo varios componentes. Para ello, se lleva a cabo un análisis factorial que permite llegar a la conclusión de que, efectivamente, todas las variables eran unidimensionales.

Se resumen los resultados en el Cuadro 10 :

Tal como era esperado, los profesores de contabilidad y auditoría enumeran que son las normas inadecuadas y el desempeño inadecuado de los auditores los principales factores para la existencia de las diferencias de expectativas.

Los ROC's, por su turno, opinan que el factor que más contribuye para las diferencias de expectativas son las ex-

Tabla 2 || Grupos de cuestiones

\begin{tabular}{|c|c|c|}
\hline & Componentes & Grupos de cuestiones \\
\hline \multirow{2}{*}{ Diferencias en el desempeño } & Desempeño inadecuado & $\begin{array}{l}\text { Comportamiento de los auditores (Grupo2) + Actuación de las } \\
\text { asociaciones y de los colegios profesionales }(G 8)=13 \text { Cuestiones }\end{array}$ \\
\hline & Normas inadecuadas & $\begin{array}{l}\text { Normas de auditoría (G1) + Limitaciones técnicas de la auditoría } \\
(\mathrm{G} 4+\text { Limitaciones de la contabilidad }(\mathrm{G} 10)=15 \text { Cuestiones }\end{array}$ \\
\hline \multirow[b]{2}{*}{ Diferencias de razonabilidad } & $\begin{array}{l}\text { Relacionadas con } \\
\text { la organización }\end{array}$ & $\begin{array}{l}\text { Comportamiento de los gestores (G3) + Gobierno de las socieda- } \\
\text { des }(G 5)+\text { Estructura de control }(G 6)=14 \text { Cuestiones }\end{array}$ \\
\hline & $\begin{array}{l}\text { Relacionadas con } \\
\text { la sociedad }\end{array}$ & $\begin{array}{l}\text { Mercado de auditoria (G7) + Comportamiento de los usuarios de la } \\
\text { información financiera (G9)+ Percepciones da la sociedad tiene de } \\
\text { la auditoria }(G 11)=15 \text { Cuestiones }\end{array}$ \\
\hline
\end{tabular}

Tabla 3 | Alfa de Cronbach

\begin{tabular}{l|c|c|c}
\multicolumn{2}{c|}{ Grupos } & Cuestiones retiradas & Alfa de Cronbach \\
Desempeño inadecuado & $\mathrm{G} 2+\mathrm{G} 8$ & $02.01 ; 02.04$ & 0,785 \\
\hline Normas inadecuadas & $\mathrm{G} 1+\mathrm{G} 4+\mathrm{G} 10$ & $01.02 ; 01.04$ & 0,751 \\
\hline $\begin{array}{l}\text { Diferencias de razonabilidad } \\
\text { relacionadas con la organización }\end{array}$ & $\mathrm{G} 3+\mathrm{G} 5+\mathrm{G} 6$ & $05.05 ; 03.01$ & 0,723 \\
\hline $\begin{array}{l}\text { Diferencias de desempeño } \\
\text { relacionadas con la sociedad }\end{array}$ & $\mathrm{G} 7+\mathrm{G} 9+\mathrm{G} 11$ & $11.09 ; 11.03 ; 11.04 ; 11.02 ; 11.01$ & 0,538 \\
\hline
\end{tabular}

1 El coeficiente de fiabilidad interna alfa de Cronbach es un método para estimar la fiabilidad. La fiabilidad es la razón entre la variancia de los factores correctos y la variancia de los valores observados. Cuando no hay errores, la variancia de los valores correctos sobre la variancia de los valores observados es $=1$. En el caso en que esta situación se produzca, la fiabilidad es perfecta.

2 Para las ciencias sociales se entiende que por encima del 0,5 la fiabilidad es adecuada. 


\begin{tabular}{|l|l|c|c|}
\hline \multicolumn{1}{|c|}{ Profesores } & \multicolumn{1}{|c|}{ ROC's } & Auditores Internos & ATC's \\
\hline Desempeño inadecuado & $\begin{array}{l}\text { Diferencias de razonabilidad } \\
\text { relacionadas con la sociedad }\end{array}$ & Desempeño inadecuado & $\begin{array}{l}\text { Diferencia de razonabilidad } \\
\text { Normasiada a la sociedad }\end{array}$ \\
\hline
\end{tabular}

Cuadro 1 Resultados

pectativas poco razonables de los usuarios de la información financiera. Similar punto de vista es compartido por los ATC's, aunque apenas en lo que se refiere a las expectativas poco razonables relacionadas con la sociedad.
Para los Al, la principal razón que provoca la existencia de las diferencias de expectativas está en el desempeño inadecuado por parte de los auditores, el cual es un resultado sorprendente.

\section{CONCEPCIÓN DE UN MODELO EXPLICATIVO DE LAS DIFERENCIAS DE EXPECTATIVAS EN AUDITORÍA PARA CADA GRUPO DE ENCUESTADOS}

En este punto, se busca elaborar un modelo explicativo de las razones más relevantes para la existencia de diferencias de expectativas, según cada uno de los grupos profesionales.

Atendiendo al cuestionario enviado, el modelo de regresión logística es el mas apropiado de utilizar, una vez quese busca obtener la visión de cada grupo en contraste con la visión de los otros. Asi, son, utilizadas como variables dependientes los profesores y los auditores (ROC's, los Al y los ATC's), variables que a continuación son analizadas.

En relación a los profesores, las variables más explicativas son Tabla $4 \mathbf{0}$.

El modelo que caracteriza su visión en relación a las diferencias de expectativas en Portugal es:

$$
\begin{gathered}
E(Y)=\left(1+\exp \left(-\beta^{\prime} X\right)\right)^{-1} \\
\beta^{\prime} X=\beta_{0}+\beta_{1} X_{1}+\beta_{2} X_{2}+\beta_{3} X_{3}+\beta_{4} X_{4} \ldots+\beta 11 X 11 \\
\text { La función respuesta logística estimada es dada por: } \\
\hat{\pi}=(1+\exp (0,13+1,576 \mathrm{~V} 0303+2,284 \mathrm{~V} 0402 \\
+1,263 \mathrm{~V} 0404+0,637 \mathrm{~V} 0407-2,365 \mathrm{~V} 0504-1,709 \\
\text { V0601-2,862 V0701 }+1,244 \mathrm{~V} 0802-1,416 \mathrm{~V} 1103 \\
-0,591 \mathrm{~V} 1104+1,721 \mathrm{~V} 1107))-1
\end{gathered}
$$

\begin{tabular}{|c|c|c|c|c|c|c|c|}
\hline & & B & S.E. & Wald & df & Sig. & $\operatorname{Exp}(B)$ \\
\hline Step & V0303 & 1,576 &, 430 & 13,448 & 1 & , 000 & 4,835 \\
\hline \multirow[t]{12}{*}{$1^{\text {a }}$} & V0402 & 2,284 & ,353 & 41,854 & 1 & ,000 & 9,812 \\
\hline & V0404 & 1,263 & ,368 & 11,782 & 1 & ,001 & 3,534 \\
\hline & V0407 & ,637 & ,356 & 3,206 & 1 & 073 & 1,891 \\
\hline & V0504 & $-2,365$ & ,675 & 12,284 & 1 & ,000 & ,094 \\
\hline & V0601 & $-1,709$ & ,415 & 16,975 & 1 & ,000 & 181 \\
\hline & V0701 & $-2,862$ & ,386 & 55,101 & 1 & ,000 & ,057 \\
\hline & V0703 &, 548 & ,346 & 2,500 & 1 & , 114 & 1,729 \\
\hline & V0802 & 1,244 & ,334 & 13,911 & 1 & ,000 & 3,469 \\
\hline & V1103 & $-1,416$ & ,439 & 10,401 & 1 & ,001 & 243 \\
\hline & V1104 &,- 591 &, 326 & 3,285 & 1 & 070 &, 554 \\
\hline & V1107 & 1,721 & ,715 & 5,799 & 1 & 016 & 5,590 \\
\hline & Constant & 130 & 875 & ,022 & 1 & 882 & 1,138 \\
\hline
\end{tabular}

Se puede concluir que la visión de los profesores está caracterizada por opinar, manteniendo todas las otras variables constantes ${ }^{3}$ :

- 9,812 veces más que los otros que el informe de auditoría es demasiado sintético;

Tabla 4 Variables in the Equation

a. variable(s) entered on step 1: V0303, V0402, V0404, V0407, V0504, V0601, V0701, V0703, V0802, V1 103, V1104, V1 107.

\footnotetext{
3 Todas las variables del modelo son explicativas son explicativas de la visión de los profesores, pero las que tienen un $\operatorname{Exp}(B)$ superior a 1 son las variables que tienen un mayor peso explicativo en el modelo.
} 
- 5,59 veces más que los otros que el grado cultural de la sociedad influencia la forma de entender y comprender el papel de la auditoría;

- 4,835 veces más que los otros que, en el comportamiento de los gestores, los objetivos individuales se sobreponen a los colectivos;

- 3,534 veces más que los otros que no existe una auditoría continúa;

- 3,469 veces más que los otros que existe insuficiente poder disciplinario por parte de las asociaciones y de los colegios profesionales;

- 1,891 veces más que los otros que existen limitaciones en la auditoría basada en el riesgo;

En relación à la visión de todos los auditores, las variables más explicativas son Tabla $5 \mathbf{0}$.

El modelo es el siguiente:

$$
\begin{gathered}
E(Y)=\left(1+\exp \left(-\beta^{\prime} X\right)\right)^{-1} \\
\beta^{\prime} X=\beta_{0}+\beta_{1} X_{1}+\beta_{2} X_{2}+\beta_{3} X_{3}+\beta_{4} X_{4} \ldots+\beta 11 X 11
\end{gathered}
$$

La función respuesta logística estimada es dada por:

$\hat{\pi}=(1+\exp (0,849+0,199 \vee 0303+0,097 \mathrm{~V} 0402$ $+0,284 \mathrm{~V} 0404+0,504 \mathrm{~V} 0407+9,922 \mathrm{~V} 0504+4,944$ $\mathrm{V} 0601+15,313 \mathrm{~V} 0701+0,290 \mathrm{~V} 0802+4,342 \mathrm{~V} 1103$ $+1,852$ V1104 $+0,171 \vee 1107))-1$

La visión de los auditores está caracterizada por pensar:

- 15,313 veces más que los profesores que los honorarios son insuficientes para ampliar el ámbito y alcance de la auditoría;

- 9,9 veces más que los profesores que los señales de alerta y prevención son poco utilizados en los informe de gestión;

- 4,9 veces más que los profesores que los consejos fiscales son poco eficientes;

- 4,3 veces más que los profesores que las diferencias de expectativas en auditoria tienen solución;

- 1,8 veces más que los profesores que la auditoria puede ser considerada un contra poder dentro de la sociedad.

\section{LA OPINIÓN DE LOS USUARIOS DE LA INFORMACIÓN FINANCIERA SOBRE EL DESARROLLO DE LA AUDITORIA}

Como se hizo referencia en el transcurso de este trabajo, existe entre los usuarios de la información financiera la noción de que los auditores son responsables por la elaboración de los estados financieros y, tal como sería de esperar, se trata de una percepción que se ha venido a confirmar en la encuesta elaborada y enviada a las empresas con cotización bursátil, a las aseguradoras y a los analistas financieros. En efecto, se puede constatar que para el $65 \%$ de las empresas y para el $62 \%$ de los analistas financieros la responsabilidad por la elaboración de los estados financieros es tanto de los administradores de las empresas como de los auditores. Se trata, pues, de una percepción errónea acerca del trabajo desarrollado por los auditores, porque la responsabilidad de éstos reside en emitir una opinión sobre si los estados financieros representan una imagen verdadera y

\begin{tabular}{|c|c|c|c|c|c|c|c|}
\hline & & B & S.E. & Wald & df & Sig. & $\operatorname{Exp}(B)$ \\
\hline Step & V0303 & $-1,616$ & 430 & 14,142 & 1 & , 000 & 199 \\
\hline \multirow[t]{11}{*}{$1^{\text {a }}$} & V0402 & $-2,332$ & 353 & 43,642 & 1 & ,000 & 097 \\
\hline & V0404 & $-1,259$ & ,365 & 11,909 & 1 & ,001 & ,284 \\
\hline & V0407 &,- 685 & ,356 & 3,693 & 1 & ,055 & ,504 \\
\hline & V0504 & 2,295 & ,663 & 11,995 & 1 & ,001 & 9,922 \\
\hline & V0601 & 1,598 & ,402 & 15,823 & 1 & ,000 & 4,944 \\
\hline & V0701 & 2,729 & ,373 & 53,604 & 1 & ,000 & 15,313 \\
\hline & V0802 & $-1,238$ & 331 & 13,970 & 1 & ,000 & 290 \\
\hline & V1103 & 1,468 & ,443 & 10,993 & 1 & ,001 & 4,342 \\
\hline & V1104 & ,616 & ,324 & 3,625 & 1 & ,057 & 1,852 \\
\hline & V1107 & $-1,766$ & 704 & 6,295 & 1 & 012 & 171 \\
\hline & Constant &,- 164 & ,865 & ,036 & 1 & ,850 & ,849 \\
\hline
\end{tabular}

Tabla 5 Variables in the Equation 
apropiada de la empresa y no reside en elaborar dichos estados. Como viene mencionado en el párrafo de las responsabilidad de la Certificação Legal de Contas - CLC (informe de auditoria) «es responsabilidad de la empresa la preparación de los estados financieros que presente de forma verdadera y apropiada la posición financiera de la empresa [...] nuestra responsabilidad (de auditores) consiste en expresar una opinión profesional e independiente basada en nuestro examen a los referidos estados financieros». Así, una de las razones para que en la mente de los usuarios de la información financiera se haya producido esta idea sobre el trabajo del auditor se debe a los servicios extra-auditoría que muchas empresas de auditoría proporcionan a sus clientes, creando la idea de que los auditores también son responsables por la preparación de los estados financieros presentados y divulgados. Como refiere Drexler (2005, p. 1617), éstos son el resultado de un conjunto de negociaciones entre la gestión y el auditor, lo que puede provocar dudas sobre la demarcación de deberes y responsabilidades entre la gestión y los auditores.

Como es referido por Mckeown et al. (1991) y DeAngelo (1981), la dimensión de la empresa de auditoría y por inherencia el peso que sus clientes tienen en el total de facturación puede poner en causa la independencia del auditor. En Portugal, el Código Deontológico de los Censores Jurados de Cuentas aconseja que el peso de la facturación de un determinado cliente no sobrepase el $15 \%$ del total de la facturación; sin embargo, deja en abierto la posibilidad de que este límite sea sobrepasado siempre que no se coloque en causa su independencia. Así, se está ante la presencia de una variable muy subjetiva que es la independencia del auditor. Cuando se enfrentan a esta cuestión, los encuestados consideran que se trata de una cuestión importante, ya que para el $86 \%$ de las empresas y para el $64 \%$ de los analistas financieros el peso de los clientes puede afectar a la imparcialidad de las pequeñas empresas de auditoría. A pesar de ello, no comparten la opinión de que las empresas de menor dimensión sean más susceptibles, de que su imparcialidad se vea afectada que en las empresas de mayor dimensión, es decir, el problema de la imparcialidad no está en la dimensión de la empresa sino más bien en el peso que los clientes tienen en esa empresa.

En relación con el desarrollo del trabajo de auditoría es de destacar que, a pesar de que el trabajo del auditor está basado en muestras, frente al volumen y complejidad actuales de las transacciones empresariales, para el $24 \%$ de las empresas cuestionadas el trabajo del auditor no se basa en muestras sino, por el contrario, sobre la totalidad de las transacciones de las empresas. Se constata con ello que, relativamente al trabajo del auditor, existe un porcentaje significativo de empresas que desconocen cómo se efectúa una auditoria.

En el análisis del papel de la auditoría en la sociedad se verifica que los encuestados juzgan que aquélla esta imbuida de un espíritu de interés público, hecho que concuerda con la teoría de dar credibilidad a la información financiera que procura explicarla con base en su interés público. A pesar de la unanimidad existente a la hora de considerar a la auditoría como estando dotada de espíritu de servicio de interés público, se detecta que, para las empresas, los escándalos financieros, en algunos de los cuales se sospecha que estén implicadas empresas de auditoría, no han hecho tambalearse la confianza de éstas en relación con el «espíritu» de la auditoría. Sin embargo, una posición contraria es asumida por los analistas financieros. En efecto, el $53 \%$ de estos encuestados considera que los auditores no defienden el interés público. Por ello, conciben que la autorregulación de la profesión es claramente insuficiente. Así, los encuestados reclaman una mayor intervención gubernamental en materia de supervisión y de regulación de la actividad de auditoría. Esta posición de los usuarios de la información financiera es opuesta a la posición defendida por los auditores e, incluso, por los propios profesores. Éstos, a pesar de que son usuarios de la información financiera, no comparten la opinión de las empresas y de los analistas financieros. Una razón para esta posición puede encontrarse en los conocimientos más profundos que poseen, relacionados con las normas de auditoría y con los organismos que tutelan la profesión auditora.

Cuando se centran en el mercado de auditoría, los usuarios de la información financiera hacen referencia a que la existencia de oligopolio de la profesión, basado en grandes empresas multinacionales, es perjudicial para una competencia libre y abierta. Esta posición no es asumida por los auditores que caracterizan el mercado portugués como siendo mayoritariamente constituido por empresas de pequeña dimensión.

A pesar de ser reconocido que los escándalos financieros que afectaron a las empresas de auditoría han tenido repercusiones negativas en la imagen de los auditores, los encuestados, unánimemente, han respondido que la profesión no ha llevado a la práctica las medidas que la sociedad exige en relación con el papel que el auditor debe desempeñar. Entre esas exigencias encontramos el papel que el auditor debe desempeñar en relación con sus responsabilidades a la hora de detectar errores y fraudes. Las normas profesionales ponen de relieve que los auditores no son responsables por la detección de errores y de fraudes: «la responsabilidad por la prevención y detección de fraudes y de errores reside en la gerencia [...] el revisor / auditor no es ni puede ser responsabilizado por la prevención de fraudes y errores» (NIR 240). Sin embargo, la sociedad exige que, en este campo, los auditores tengan un papel más interventor y que asuman mayores responsabilidades. En efecto, el $87 \%$ de los analistas financieros y el $71 \%$ de las empresas considera que las responsabilidades de los auditores en este punto deben ser ampliadas y que dicho aumento de responsabilidades deberá pasar por una búsqueda activa de indicios de fraude. Estas conclusiones no se restringen sólo a Portugal, ya que varios estudios, como es el caso de la Cohen Comisión (1976) y Steen (1991)4, demostraron que los usuarios de la información financiera esperan de los auditores en relación con esta área más que lo que ellos efectivamente les proporcionan. Como ya se habia analizado al principio de este capítulo, las empresas respondieron con una media de 2,6 que la detección de errores y de fraudes es la cuestión acerca 
de la cual las diferencias de expectativas son más acentuadas. De igual modo, los analistas financieros eligieron también la detección de fraudes con una media de 1,75.

En lo que respecta a la continuidad de la empresa, no se encontraron indicios de que la mayoría de los usuarios de la información financia tenga expectativas poco razonables. A pesar de ello, relieves de resaltar que este estudio presenta conclusiones semejantes a las de estudios realizados en $\mathrm{Ca}$ nadá (McDonald Comission) y en Gran Bretaña (KPMG). De hecho, un elevado porcentaje de encuestados, el 38\% en el sector de las empresas, considera que la falta de referencia a cualquier problema relacionado con la continuidad de la empresa en la CLC significa que el auditor garantiza que ésta va a continuar en funcionamiento durante los próximos años. Esta posición de los usuarios de la información financiera es antagónica a lo estipulado en la NIR 570 que es bien clara al respecto: «el revisor / auditor no puede predecir acontecimientos o condiciones futuras que puedan dar origen a que una entidad deje de proseguir en continuidad. Consecuentemente, la falta de cualquier referencia a la incertidumbre sobre la continuidad en el informe de revisión / auditoría no puede ser vista como una garantía de la capacidad de la entidad para proseguir en continuidad». Otra conclusión que se puede extraer en relación con la problemática de la continuidad, se refiere al hecho de que los usuarios de la información financiera no consideren que un auditor al emitir una opinión con reservas sobre la continuidad de una empresa esté precipitando la quiebra de la misma. Esta posición es la opuesta a la que defendieron Elliot y Jacobson (1987) y Psaros y Zhang (1994) quienes, argumentan que un auditor al pronunciarse sobre la eventual discontinuidad de la empresa está precipitando su quiebra, dando origen a la llamada self-fulfilling prophecy. También la posición defendida por DeFond (1992) y por Schwartz y Menon (1985) no es soportada por los datos que se obtienen en este trabajo. En efecto, el $80 \%$ de las empresas encuestadas no ponderaría la posibilidad de cambiar de auditor en el caso de que éste pusiese en causa la continuidad de la empresa.

En lo que se refiere al objetivo de la auditoría de verificar si los estados financieros de las empresas han sido elaborados de acuerdo con los principios contables generalmente aceptados y si se corresponden con una imagen verdadera y apropiada, ello es algo reductor para los usuarios de la información financiera. En efecto, una gran parte de los encuestados hace referencia a que la auditoría debería extender su campo de actuación hacia otras fronteras más allá de las estrictamente financieras. Así, a pesar de que la mayor parte de los encuestados considera que el informe de auditoría es un instrumento importante en el proceso de toma de decisión (81\% de los analistas financieros, $86 \%$ de las empresas), destaca también que la información objeto de auditoría debería ser más abarcadora, englobando la responsabilidad social de prestación de cuentas ( $60 \%$ de los analistas financieros, $71 \%$ de las empresas) y la información prospectiva de la empresa (66\% de los analistas, $71 \%$ de las empresas), relegando para un segundo plano la información histórica. Como corolario, los usuarios de la información financiera sostienen que el actual informe de auditoría, de contenido estrictamente financiero, es insatisfactorio.

Tal como es referido por la Cohen Commission (1976) y por el Libro Verde de la UE (1996), se constata que los usuarios de la información financiera portuguesa también juzgan que el informe de auditoría no expresa la totalidad del trabajo de auditoría desarrollado y están también en la línea del estudio de Anderson y Epstein (1995) $)^{5}$ en que verificaron que el 19,5\% de los encuestados tenía dificultades a la hora de leer el informe de auditoría. En Portugal, dicho porcentaje se eleva para el 40 y $38 \%$, respectivamente, en relación con los analistas financieros y con las empresas.

De acuerdo con las normas de auditoría DRA 700 «[...] examen destinado a proporcionar una seguridad aceptable que le permita al revisor/auditor expresar, de una forma positiva, su opinión sobre si tales estados financieros tomados como un todo están, o no, exentos de distorsiones materialmente relevantes» $y$, contrariamente a lo referido por Boyd et al. (2001), los usuarios de la información financiera consideran que por el hecho de que el informe de auditoría no mencione cualquier reserva, esta situación no debe ser interpretada como una garantía de que la información financiera producida y divulgada por la empresa sea correcta. De igual modo, cuando se produce la quiebra de una empresa sometida a auditoría, el hecho de que esto ocurra no significa que el auditor haya ejecutado su trabajo de una forma incorrecta. Esta conclusión era en cierto modo la esperada. ya que, los usuarios de la información financiera son conscientes de algunas de las limitaciones de la auditoría, sobre todo, en lo que se relaciona con las formas de enjuiciar que tienen por base técnicas de muestreo.

Además de una mayor intervención gubernamental en aspectos de supervisión y reglamentación de la actividad profesional, los usuarios de la información financiera refieren, de igual modo, que la implementación de un eficaz gobierno de las sociedades y la implementación de comités de auditoría podrían ser medidas que contribuyesen a la reducción de las diferencias de expectativas en auditoría.

Así, se concluye, al contrario de los estudios citados, que las expectativas de los usuarios de la información financiera no son tan irrealistas como sucede en otros países. Se exige una mayor intervención en materia de fraudes porque, como es observado, esta situación es la que se apunta como siendo una de las cuestiones en relación con la cual las diferencias de expectativas son más acentuadas. Se considera, asimismo, que el informe de auditoría es demasiado sintético y que, además de aspectos meramente financieros, debería abarcar no sólo aspectos de carácter social sino también poner de relieve la información prospectiva de la empresa.

Para la mayor parte de los usuarios de la información financiera, la auditoría es vista como un estabilizador de las relaciones financieras y económicas de la sociedad. 


\section{CONCLUSIONES}

Las diferencias de expectativas son una realidad en Portugal. De hecho, a lo largo del trabajo se deja constancia de que la sociedad portuguesa exige una actuación más eficiente por parte de los auditores, sobre todo en lo que respecta a materias relacionadas con el fraude y con su comunicación. Sin embargo, no se puede dejar de subrayar que los usuarios portugueses de la información financiera poseen, igualmente, algunas ideas erróneas sobre el papel que los auditores pueden, razonablemente, desempeñar y asegurar.

Las razones aducidas a la hora de justificar la existencia de diferencias de expectativas varía de grupo para grupo. Por un lado, los auditores, predominantemente, le atribuyen a la sociedad y a la organización de las empresas las razones de su existencia, mientras que los usuarios de la información financiera, por su parte, enumeran las normas inadecuadas y el desempeño inadecuado como razones para la existencia de diferencias de expectativas.

Por otro lado, al contrario de lo que ocurre en otros países, en Portugal las normas de auditoría no son un componente importante de la problemática de las diferencias de expectativas. Sin embargo, es de subrayar que los encuestados reclaman una mayor intervención gubernamental en la elaboración de normativas de auditoría, lo que puede ser indicio claro de que existe alguna excesiva autoreglamentación de la profesión.

\section{Referências}

BARON, C. et al, Uncovering corporate irregularities: are we closing the expectation gap?. Journal of Accountancy, New York, v. 144. October 1997.

BOYD, D. et al. The audit report: a misunderstanding gap between users and preparers. National Public Accountant, Washington, p. 56-60, January 2001.

DE ANGELO, L. Auditor Size and Audit Quality. Journal of Accounting and Economics, p. 183-199, 1981.

DE FOND, M. The association between changes in client firm agency costs and auditor switching. Auditing: A journal of practice and theory, New York, Spring, p. 16-31, 1992.

DREXLER, P. Can proposed audit adjustments challenge auditor independence. PA Journal, August 2005.

ELLIOT, R.; JACOBSON, P. The auditor's standard report: The last word or in need of change?.Journal of Accountancy, New York, n. 165, p. $72-79,1987$

GODSELL, D. Legal liability and the audit expectation gap. Singapore Accountant, Singapore, v. 8, p. 25-28, November 1992.

HUMPHREY. et al. Protecting against detection: The case of auditors and fraud?. Accounting, Auditing e Accountability Journal, Londres, v. 6, n. $1,1993 \mathrm{~b}$.

The audit expectation gap in Britain: an empirical investigation. Accounting and Business Research, London, v. 23, p. 359-411, Summer, 1993a.

LOWE, A. The auditor's detection responsibility: is there an expectation gap?. Journal of Accountancy, New York 1980.

MCENROE, J.; MARTENS, S. Auditors' and investors' perceptions of the expectation gap. Accounting Horizons, Sarasota, v. 15, n. 4, December 2001.

MCKEOWN, J. et al. Towards an explanation of auditor failure to modify the audit opinions of bankrupt companies. Auditing: A Journal of Practice \& Theory 10, New York, n. 10, 1991. (Supplement), p. 1-13.

PSAROS, J.; ZHANG, M. The going concern audit opinion: Australian evidence. Perspectives on Contemporary Auditing, p. 39-46, 1994. ROEBUCK, P. et al. Understanding assurance services reports: a user perspective. Accounting and Finance, Clayton, p. 214, November 2000 SCHWARTZ, K.; MENON, K. 1985, Auditors switches by failing finns. The Livro Verde, 1996.

\section{NOTA - Endereço dos autores}

Escola Superior de Gestão de Tomar Instituto Politécnico de Tomar Estrạda da Serra - Quinta do Contador Tomar - Portugal 2300-313
Universidad Complutense de Madrid

Faculdad de Ciencias Económicas y Empresariales

Edificio Prefabricado, despacho 332 - norte

Campus de Somosaguas - Pozuelo de Alarcón

Madrid - España

28223 